\title{
THE CONVERSE OF THE INTERMEDIATE VALUE THEOREM: FROM CONWAY TO CANTOR TO COSETS AND BEYOND
}

\author{
GREG OMAN
}

\begin{abstract}
The classical Intermediate Value Theorem (IVT) states that if $f$ is a continuous real-valued function on an interval $[a, b] \subseteq \mathbb{R}$ and if $y$ is a real number strictly between $f(a)$ and $f(b)$, then there exists a real number $x \in(a, b)$ such that $f(x)=y$. The standard counterexample showing that the converse of the IVT is false is the function $f$ defined on $\mathbb{R}$ by $f(x):=\sin \left(\frac{1}{x}\right)$ for $x \neq 0$ and $f(0):=0$. However, this counterexample is a bit weak as $f$ is discontinuous only at 0 . In this note, we study a class of strong counterexamples to the converse of the IVT. In particular, we present several constructions of functions $f: \mathbb{R} \rightarrow \mathbb{R}$ such that $f[I]=\mathbb{R}$ for every nonempty open interval $I$ of $\mathbb{R}(f[I]:=\{f(x): x \in I\})$. Note that such an $f$ clearly satisfies the conclusion of the IVT on every interval $[a, b]$ (and then some), yet $f$ is necessarily nowhere continuous! This leads us to a more general study of topological spaces $X=(X, \mathcal{T})$ with the property that there exists a function $f: X \rightarrow X$ such that $f[O]=X$ for every nonvoid open set $O \in \mathcal{T}$.
\end{abstract}

\section{INTRODUCTION}

Shortly after exposure to continuity, most calculus students are introduced to the Intermediate Value Theorem (IVT).

Theorem 1 (Intermediate Value Theorem). Let $[a, b]$ be any real interval and suppose that $f:[a, b] \rightarrow \mathbb{R}$ is a continuous function. If $y$ is any real number strictly between $f(a)$ and $f(b)$, then there exists $x \in(a, b)$ such that $f(x)=y$.

The IVT has several interesting theoretical applications. To mention but one, it enables one to prove the existence of roots of certain equations which often cannot be solved algebraically. In fact, many standard calculus texts enable professors to challenge differential calculus students by including several such proofs as exercises (for example, Prove that the equation $\cos (x)$ $=x$ has a solution in $(0,1)$ using the Intermediate Value Theorem appears as problem \#41 in Stewart [18]). 


\section{CONVERSE OF THE INTERMEDIATE VALUE THEOREM}

At first glance, it may seem that the IVT has a sort of converse. We, consider the following question.

Question 1. Suppose that $f$ is a real-valued function defined on an interval I. Assume further that for any two real numbers $x_{1}<x_{2}$ in I: if $y$ is a real number strictly between $f\left(x_{1}\right)$ and $f\left(x_{2}\right)$, then there exists a real number $x \in\left(x_{1}, x_{2}\right)$ such that $f(x)=y$ (such a function $f$ is said to satisfy the intermediate value property on I). Must $f$ be continuous on I?

It is not too hard to see that the answer is 'no', and the following function $f: \mathbb{R} \rightarrow \mathbb{R}$ is a standard counterexample ${ }^{1}$

$$
f(x):= \begin{cases}\sin \left(\frac{1}{x}\right), & \text { if } x \neq 0 \\ 0, & \text { if } x=0\end{cases}
$$

Although the function $f$ defined above certainly proves that Question 1 has a negative answer, there is a sense in which the counterexample is a bit weak. In particular, $f$ is discontinuous only at $x=0$. On the other hand, the negative answer to Question 1 also raises another question. Are there "natural" conditions one might impose on a function $f$ that satisfies the intermediate value property which guarantee that $f$ is continuous? It turns out that the answer is 'yes'. We mention one example (see Sohrab [17], Proposition 4.5.1 for a proof; we refer the reader to the same source for additional examples).

Proposition 1. Suppose that $f: \mathbb{R} \rightarrow \mathbb{R}$ is injective and satisfies the intermediate value property on $\mathbb{R}$. Then $f$ is continuous on $\mathbb{R}$.

We end our digression by noting the following interesting fact. Recall that the (standard) completeness axiom $(C)$ for the reals is the statement that every nonempty subset of $\mathbb{R}$ which is bounded above has a least upper bound. Consider removing this axiom from the standard list of axioms for the complete ordered field $\mathbb{R}$ of real numbers. Then one is left with the set $\mathcal{A}$ of ordered field axioms, and we have the following proposition.

Proposition 2. The completeness axiom $(C)$ and the Intermediate Value Theorem are equivalent in $\mathcal{A}$ (that is, $\mathcal{A}+(C)$ proves IVT and $\mathcal{A}+I V T$ proves $(C)$ ).

\footnotetext{
${ }^{1}$ Other counterexamples can be obtained via Darboux's Theorem. To mention but one, let $f$ be defined on $\mathbb{R}$ by

$$
f(x):= \begin{cases}x^{2} \sin \left(\frac{1}{x}\right), & \text { if } x \neq 0 \\ 0, & \text { if } x=0 .\end{cases}
$$

Then $f$ is differentiable on $\mathbb{R}$, yet $f^{\prime}$ is not continuous at 0 . However, Darboux's Theorem yields that $f^{\prime}$ satisfies the intermediate value property on $\mathbb{R}$.
}

MISSOURI J. OF MATH. SCI., FALL 2014 


\section{G. OMAN}

We refer the reader to Teismann [19] for a proof of the previous proposition and for a sampling of other statements equivalent (in $\mathcal{A}$ ) to $(\mathrm{C})$.

We now focus our efforts on producing stronger counterexamples to the converse of the IVT. In particular, we study functions $f: \mathbb{R} \rightarrow \mathbb{R}$ with the property that

$$
\begin{aligned}
& (\star) f[I]=\mathbb{R} \text { for every nonempty open interval } \\
& I \subseteq \mathbb{R}(f[I]:=\{f(x): x \in I\}) .
\end{aligned}
$$

It is not immediately obvious that such a wacky function $f$ should even exist (but it does!). For now, we ask the reader to take $f$ 's existence on faith. Any such $f$ clearly satisfies the intermediate value property on $\mathbb{R}$. However (as we will prove in the next section), any $f$ satisfying $(\star)$ is not only discontinuous at some real number $x$, but discontinuous at every real number $x$. Thus, $f$ in a sense is a very strong counterexample to the converse of the IVT.

The outline of this article is as follows. Our first goal is to present several folklore methods for creating functions $f: \mathbb{R} \rightarrow \mathbb{R}$ which satisfy $(\star)$ (henceforth, we refer to such functions as $(\star)$-functions for brevity). We present constructive, topological, and algebraic methods for establishing the existence of $(\star)$-functions. We are then naturally lead to study a more general topological notion defined as follows. Let $X=(X, \mathcal{T})$ be a topological space. Say that $X$ is a $(\star)$-space provided there exists a function $f: X \rightarrow X$ with the property that $f[O]=X$ for every nonempty open set $O \in \mathcal{T}$. An obvious necessary condition for $X$ to be a $(\star)$-space is that every nonempty open subset of $X$ has the same cardinality as $X$. On the other hand, we prove that if in addition there is a base $\mathcal{B}$ for the topology $\mathcal{T}$ such that $|\mathcal{B}| \leq|X|$, then $X$ is a $(\star)$-space (in fact, we prove a result which is a bit more general than this). We conclude the paper by showing that this additional condition is not necessary, but that if it is dropped, then $X$ need not be a $(\star)$-space.

\section{BASE $13 ? !$}

Professor John Conway of Princeton University is well-known for his many creative and diverse mathematical discoveries. We pause to list a few of them. In game theory, he invented The Game of Life and The Surreal Numbers ${ }^{2}$. He made a fundamental contribution to the theory of finite groups by discovering three of the 26 sporadic groups. Lastly, we

\footnotetext{
${ }^{2}$ Donald Knuth coined the term "surreal number." In fact, his novelette Surreal Numbers (Knuth [11]) was published before Conway published his discovery. Knuth's book was written not to formally describe Conway's theory, but to give an allegory of how mathematical discoveries are made.
} 


\section{CONVERSE OF THE INTERMEDIATE VALUE THEOREM}

mention his so-called Free Will Theorem ${ }^{3}$ (joint with Simon Kochen) in theoretical physics. Possibly less well-known is his constructive example of a $(\star)$-function using base 13 representations of real numbers. We will discuss his idea shortly.

As noted in the previous section, any $(\star)$-function clearly satisfies the intermediate value property. However, every $(\star)$-function is necessarily discontinuous at every real number, as we now prove.

Proposition 3. Suppose that $f: \mathbb{R} \rightarrow \mathbb{R}$ is a ( $\star$ )-function. Then $f$ is discontinuous at every real number.

Proof. Assume that $f: \mathbb{R} \rightarrow \mathbb{R}$ is a $(\star)$-function. Suppose by way of contradiction that there exists $x \in \mathbb{R}$ such that $f$ is continuous at $x$. Let $V:=B_{1}(f(x))$ be the open ball of radius one centered at $f(x)$. Then there exists an open neighborhood $U$ of $x$ such that $f[U] \subseteq V$. Since $f$ is a $(\star)$ function, it is clear that $f[U]=\mathbb{R}$. But then $\mathbb{R} \subseteq V$, and we have obtained a contradiction.

We have yet to show that a $(\star)$-function exists. We now work to remedy this deficiency. Consider the distinct "base 13 symbols"

$$
0,1,2,3,4,5,6,7,8,9, A, B, C
$$

(think of $A$ (in base 10) as 10, $B$ as 11, and $C$ as 12). Every real number $r \in[0,1)$ can be expressed in base 13 as follows.

$$
r=c_{1} c_{2} c_{3} \ldots c_{n} \ldots
$$

where each $c_{i} \in\{0,1,2,3,4,5,6,7,8,9, A, B, C\}$. Moreover, we may assume that for every $n>0$, there exists $m>n$ such that $c_{m} \neq C$ (in other words, the base 13 representation of $r$ does not end in a sequence of $C \mathrm{~s}$ ). Let us call such a representation of $r$ a normal representation. It is well-known that $r$ has a unique normal representation (in any base). A tail of $r$ (with the notation of (2.1 above) $)$ is a sequence $c_{j} c_{j+1} c_{j+2} \ldots(j \geq 1)$.

We are almost ready to define Conway's Base 13 Function. Say that a tail (we modify the dummy variables for convenience) $x_{0} x_{1} x_{2} \ldots x_{n} y_{0} y_{1} y_{2} \ldots$ of $r \in[0,1)$ is special provided the following conditions hold.

(i) $x_{0}=B$ or $x_{0}=C$,

(ii) $y_{0}=A$, and

(iii) For $i>0, x_{i}$ and $y_{i}$ lie in the set $\{0,1,2,3,4,5,6,7,8,9\}$.

It is easy to see that not every element of $[0,1)$ possesses a base 13 special tail. The real number $.111 \ldots$ is such an example. However, if a base 13

\footnotetext{
[8].

${ }^{3}$ This result is not without controversy; see Goldstein, Tausk, Tumulka, and Zanghi
} 


\section{G. OMAN}

representation of $r \in[0,1)$ does possess a special tail, then it is easy to see that it is unique.

Lemma 1. Let.$c_{1} c_{2} c_{3} \ldots$ be a normal base 13 representation of $r \in[0,1)$. Then $r$ has at most one special tail.

We now introduce Conway's Base 13 Function $f$ on $[0,1)$ as follows. Let $x \in[0,1)$ and suppose $x=. c_{1} c_{2} c_{3} \ldots$ is a normal base 13 representation of $x$. Define $f$ by

$$
f(x)=\left\{\begin{array}{l}
0 \quad \text { if no tail of } x \text { is special, } \\
x_{1} x_{2} \ldots x_{n} . y_{1} y_{2} \ldots \\
\quad \text { if } x_{0} x_{1} x_{2} \ldots x_{n} A y_{1} y_{2} \ldots \text { is a special tail and } x_{0}=B, \\
-x_{1} x_{2} \ldots x_{n} \cdot y_{1} y_{2} \ldots \\
\quad \text { if } x_{0} x_{1} x_{2} \ldots x_{n} A y_{1} y_{2} \ldots \text { is a special tail and } x_{0}=C .
\end{array}\right.
$$

It is important to note that the outputs of $f$ are in base 10. Note also that $f$ is well-defined by Lemma 1 . We now show that $f$ has property $(\star)$ on $[0,1)$. This is stated in the following lemma.

Lemma 2. Let $f$ be defined on $[0,1)$ as above. Then $f[I]=\mathbb{R}$ for any open interval $I$.

Proof. Let $I=(a, b), 0 \leq a<b<1$, and let $a=. a_{1} a_{2} a_{3} \ldots$ and $b=. b_{1} b_{2} b_{3} \ldots$ be normal base 13 representations of $a$ and $b$. Now let $i$ be least such that $a_{i}<b_{i}$ (note that $0<1<2<3<4<5<$ $6<7<8<9<A<B<C)$ and let $j>i$ be least such that $a_{j}<C$ (such a $j$ exists since we are working with normal representations). Consider an arbitrary non-negative base 10 real number $x:=$ $x_{1} x_{2} \ldots x_{n} . y_{1} y_{2} y_{3} \ldots$, and consider the real number $c$ defined in base 13 by $c:=a_{1} a_{2} a_{3} \ldots a_{i} C_{i+1} C_{i+2} \ldots C_{j} B x_{1} x_{2} \ldots x_{n} A y_{1} y_{2} y_{3} \ldots$ (here $C_{i}=$ $\left.C_{i+1}=C_{i+2}=\ldots=C_{j}=C\right)$. It is easy to see that $a<c<b$. Further, it follows by definition of $f$ that $f(c)=x$. Similarly, suppose we modify $c$ by changing $x_{0}=B$ to $x_{0}=C$ and obtain the new number $c^{\prime}$. Then $a<c^{\prime}<b$ and $f\left(c^{\prime}\right)=-x$. Therefore, $f[I]=\mathbb{R}$ and the proof is complete.

We now extend $f$ to a function $\bar{f}: \mathbb{R} \rightarrow \mathbb{R}$ by defining

$$
\bar{f}\left( \pm x_{1} x_{2} x_{3} \ldots x_{n} . y_{1} y_{2} y_{3} \ldots\right):=f\left(. y_{1} y_{2} y_{3} \ldots\right) \text {. }
$$

Using the previous lemma, the following theorem follows easily (we omit the proof).

Theorem 2. The function $\bar{f}$ is a (*)-function. 


\section{CONVERSE OF THE INTERMEDIATE VALUE THEOREM}

We conclude this section with a couple of comments. First, what is spectacular about Conway's Base 13 function is that it can be defined without the axiom of choice. Since the reals can be constructed in ZF and since decimal representations can also be defined in $\mathrm{ZF}$, it follows that one can prove the existence of a $(\star)$-function without the axiom of choice. Considering how pathological $(\star)$-functions are, the fact that they can be constructed without choice is somewhat surprising. Second, one may ask what is special about using the number 13 as the base (aside from it being unlucky). The answer is 'nothing'. It simply allows us to define a function in a natural way so that the outputs are in the more familiar base 10 .

\section{Enter Cantor}

Having given a constructive example of a $(\star)$-function in the previous section, we now focus on less constructive approaches (but which yield shorter proofs). In this section, we employ some standard properties of Cantor sets. ${ }^{4}$ The ternary Cantor set has some remarkable topological properties (we refer the interested reader to Munkres [13, p. 178], Jech [10, p. 37], and [15, p. 309]). We will need only some of them. This set has measure 0 , it is closed in $\mathbb{R}$, and it is uncountable.

We now present another proof of the existence of a $(\star)$-function using Cantor sets. Let $\mathcal{S}$ denote the collection of all nonvoid open intervals with rational endpoints. Then clearly $\mathcal{S}$ is countable. Thus we may enumerate $\mathcal{S}$ as follows.

$$
\mathcal{S}:=\left\{\left(r_{1}, s_{1}\right),\left(r_{2}, s_{2}\right),\left(r_{3}, s_{3}\right), \ldots,\right\} .
$$

We now claim that there exists a collection $\mathcal{C}:=\left\{C_{1}, C_{2}, C_{3}, \ldots\right\}$ of Cantor sets with the following properties.

(a) Each $C_{i} \subseteq\left(r_{i}, s_{i}\right)$, and

(b) $C_{i} \cap C_{j}=\varnothing$ for $i \neq j$.

To prove this, we begin by letting $C_{1}$ be any Cantor set contained in $\left(r_{1}, s_{1}\right)$ (simply construct $C_{1}$ in any nontrivial closed interval $I \subseteq\left(r_{1}, s_{1}\right)$ ). Now suppose that Cantor sets $C_{1}, C_{2}, \ldots, C_{n}$ satisfying (a) and (b) above have been chosen. We define $C_{n+1}$ as follows. $C_{i}$ has measure 0 for each $i$, $1 \leq i \leq n$, hence so does $C_{1} \cup C_{2} \cup \ldots \cup C_{n}$. The interval $\left(r_{n+1}, s_{n+1}\right)$ is of positive measure, hence $\left(r_{n+1}, s_{n+1}\right) \nsubseteq\left(C_{1} \cup C_{2} \cup \ldots \cup C_{n}\right)$. Let

$X:=\left(r_{n+1}, s_{n+1}\right) \cap C_{1}^{c} \cap C_{2}^{c} \cap \ldots \cap C_{n}^{c} \neq \varnothing($ all complements are taken in $\mathbb{R})$.

${ }^{4}$ Despite the moniker, the Cantor sets were first discovered in the 1870 's by Henry Smith [16].

MISSOURI J. OF MATH. SCI., FALL 2014 


\section{G. OMAN}

Each $C_{i}$ is closed in $\mathbb{R}$, hence $C_{i}^{c}$ is open in $\mathbb{R}$. Therefore $X$ is open. Choose any $x \in X$ and let $\epsilon>0$ be such that $B_{\epsilon}(x) \subseteq X$. Finally, choose any Cantor set $C_{n+1} \subseteq B_{\epsilon}(x)$.

Now, for each $i>0$, let $f_{i}: C_{i} \rightarrow \mathbb{R}$ be a surjection $\left(f_{i}\right.$ exists because $\left.\left|C_{i}\right|=|\mathbb{R}|\right)$ and define $f: \mathbb{R} \rightarrow \mathbb{R}$ as follows.

$$
f(x)= \begin{cases}f_{i}(x), & \text { if } x \in C_{i} \text { for some } i>0 \\ 0, & \text { otherwise. }\end{cases}
$$

We prove the following theorem.

Theorem 3. The function $f$ defined above is a $(\star)$-function.

Proof. Note first that $f$ is well-defined since the sets $C_{i}$ are pairwisedisjoint. Now let $a$ and $b$ be real numbers with $a<b$, and set $I:=(a, b)$. We will prove that $f[I]=\mathbb{R}$. To see this, simply note that $I$ contains some interval $\left(r_{n}, s_{n}\right)$. Thus by the above construction, $C_{n} \subseteq\left(r_{n}, s_{n}\right) \subseteq I$. We conclude that $\mathbb{R}=f_{n}\left[C_{n}\right]=f\left[C_{n}\right] \subseteq f[I] \subseteq \mathbb{R}$. Hence, $f[I]=\mathbb{R}$, and the proof is complete.

\section{A Proof From "The BooK"}

In the present section, we present "the book" ${ }^{5}$ proof of the existence of $(\star)$-functions on $\mathbb{R}$. Specifically, we give a very short argument using only fundamental properties of cosets along with the fact that the set $\mathbb{Q}$ of rational numbers is dense in $\mathbb{R}$.

Toward this end, if $G$ is a group and $H$ is a subgroup of $G$, then for any $g \in G$, the set $H g:=\{h g: h \in H\}$ is called a right coset of $H$ in $G$. We denote the cardinality of the set $\{H g: g \in G\}$ by $[G: H]$ (called the index of $H$ in $G$ ). We now pause to record two fundamental properties of cosets. The following results can be found in most standard abstract algebra texts; see Hungerford [9] or Lang [12], for example.

Fact 1. Let $G$ be a group and let $H$ be a subgroup of $G$.

(1) For any $x, y \in G: H x=H y$ if and only if $x y^{-1} \in H$.

(2) (Lagrange) $|G|=|H|[G: H]$.

We need one simple lemma before presenting our final construction of a $(\star)$-function on $\mathbb{R}$.

\footnotetext{
${ }^{5}$ Proofs from THE BOOK [1] is a book of mathematical proofs written by Martin Aigner and Günter Ziegler. The book is dedicated to the mathematician Paul Erdös, who often referred to "The Book" in which God keeps the most elegant proof of each mathematical theorem. Legend has it that during a lecture in 1985, Erdös said, "You don't have to believe in God, but you should believe in The Book."
} 
Lemma 3. The groups $\mathbb{R}$ (under addition) and $\mathbb{R} / \mathbb{Q}$ have the same cardinality.

Proof. By $(2)$ of Fact 1 , we see that $|\mathbb{R}|=|\mathbb{Q}|[\mathbb{R}: \mathbb{Q}]=|\mathbb{Q}||\mathbb{R} / \mathbb{Q}|$. Basic cardinal arithmetic (for example, see $[7$, p. 164]) yields that $|\mathbb{R}|=|\mathbb{Q}||\mathbb{R} / \mathbb{Q}|=$ $\max (|\mathbb{Q}|,|\mathbb{R} / \mathbb{Q}|)$. We conclude that $|\mathbb{R}|=|\mathbb{R} / \mathbb{Q}|$.

We now present a strikingly simple construction of a $(\star)$-function as follows. Let $g: \mathbb{R} / \mathbb{Q} \rightarrow \mathbb{R}$ be a surjection (the existence of $g$ is guaranteed by the previous lemma), and define $h: \mathbb{R} \rightarrow \mathbb{R} / \mathbb{Q}$ by $h(r):=\mathbb{Q}+r$ (since $\mathbb{R}$ is an abelian group under addition, we switch to additive notation for cosets). Finally, let $f:=g \circ h$. Then, we have the following theorem.

Theorem 4. The function $f$ is $a(\star)$-function.

Proof. Let $a<b$, and set $I:=(a, b)$. As in the proof of Theorem 3, we show that $f[I]=\mathbb{R}$. Toward this end let $r \in \mathbb{R}$ be arbitrary. Since $g$ is onto $\mathbb{R}$, there exists some $x \in \mathbb{R}$ such that $g(\mathbb{Q}+x)=r$. We claim that

$$
f[\mathbb{Q}+x]=\{r\}
$$

To mitigate any confusion, note that the coset $\mathbb{Q}+x$ is an element of the domain of $g$, whereas $\mathbb{Q}+x$ is a subset of the domain of $h$. Let $q \in \mathbb{Q}$ be arbitrary. Then $f(q+x)=g(h(q+x))=g(\mathbb{Q}+(q+x))=g(\mathbb{Q}+x)=r$ (note that $\mathbb{Q}+(q+x)=\mathbb{Q}+x$ by (1) of Fact 1 ). This establishes (4.1). To finish the proof, observe that $\mathbb{Q}+x$ is dense in $\mathbb{R}$. In particular, there exists $\alpha \in(\mathbb{Q}+x) \cap I$. Hence (by (4.1)) $f(\alpha)=r$ and the proof is complete.

\section{5. $\star$ (Space) Power}

In the previous three sections, we concerned ourselves with finding various ways to construct $(\star)$-functions $f: \mathbb{R} \rightarrow \mathbb{R}$. Note trivially that if $f: \mathbb{R} \rightarrow \mathbb{R}$ is a $(\star)$-function, then $f[O]=\mathbb{R}$ for every nonempty open subset $O$ of $\mathbb{R}$ (since $O$ contains a nonempty open interval). This leads to the following open-ended question. What can be said of general topological spaces $X=(X, \mathcal{T})$ for which there exists a function $f: X \rightarrow X$ with the property that $f[O]=X$ for every nonempty $O \in \mathcal{T}$ ? Extending the real-valued definition, we shall henceforth call such an $f$ a $(\star)$-function. For brevity (and to fit naturally with the "star notation" throughout the paper), let us call such a topological space $X$ a $(\star)$-space.

As we have shown, $\mathbb{R}$ (with the standard topology) is a $(\star)$-space. However, the three constructions we presented of $(\star)$-functions hinged on special algebraic/topological properties of the real line. Specifically, the base 13 construction depends on the existence of decimal representations of the real numbers, the standard proof of which utilizes the completeness property of $\mathbb{R}$. The Cantor set construction uses the fact that $\mathbb{R}$ is second-countable, 


\section{G. OMAN}

and the coset construction exploits the additive group structure of $\mathbb{R}$. Thus, the following question arises naturally: if $X=(X, \mathcal{T})$ is an infinite $(\star)$ space, are there stringent topological properties that are forced on $X$ ? Of course, this question is rather imprecise, but we will show (perhaps surprisingly) that there is a sense in which the answer is 'no'. An obvious necessary condition for $X$ to be a $(\star)$-space is that every nonempty open subset of $X$ must have the same cardinality as $X$ (we state this as a lemma below). If in addition there is a basis $\mathcal{B}$ for $\mathcal{T}$ of size at most $|X|$, then we will prove that $X$ is a $(\star)$-space (we actually prove something a bit more general than this). We also show that this additional property is not necessary to guarantee that $X$ is a $(\star)$-space. On the other hand, we prove by example that it cannot be dropped entirely.

Lemma 4. Let $X$ be a $(\star)$-space. Then every nonempty open subset $O$ of $X$ has the same cardinality as $X$.

As in the literature, we call a space $X$ satisfying the conclusion of the above lemma cardinal-homogeneous (card-homogeneous for short). We now easily characterize the finite $(\star)$-spaces.

Corollary 1. Let $X=(X, \mathcal{T})$ be a finite topological space. Then $X$ is a $(\star)$-space if and only if $X$ is indiscrete (i.e. the topology $\mathcal{T}$ is trivial).

Proof. Suppose that $X=(X, \mathcal{T})$ is a finite topological space. Assume first that $X$ is a $(\star)$-space. If $O \in \mathcal{T}$ is nonempty, then by Lemma 4 , $|O|=|X|$. Since $X$ is finite, we deduce that $O=X$, and hence $X$ is indiscrete. Conversely, every indiscrete space $X$ is trivially a $(\star)$-space (since the identity map on $X$ is a ( $\star$ )-function).

We now pause to recall some relevant terminology from the literature. Let $X=(X, \mathcal{T})$ be a nonempty topological space. The dispersion character of $X$, denoted $\Delta(X)$, is defined to be the smallest cardinality of a nonempty open subset of $X$ (that is, $\Delta(X):=\min \{|O|: \varnothing \neq O \in \mathcal{T}\}$ ). Now let $\kappa$ be a cardinal. Then $X$ is said to be $\kappa$-resolvable ${ }^{6}$ if and only if there exist $\kappa$ many pairwise-disjoint dense subsets of $X$. Finally, $X$ is maximally resolvable if and only if $X$ is $\Delta(X)$-resolvable ${ }^{7}$. We present an example to illustrate the above definitions.

Example 1. Consider $\mathbb{R}$ with the standard topology. Then

(1) $\Delta(\mathbb{R})=2^{\aleph_{0}}$.

(2) $\mathbb{R}$ is maximally resolvable.

\footnotetext{
${ }^{6}$ This terminology was introduced by Ceder [3]

${ }^{7}$ It is easy to see that if $X$ is $\kappa$-resolvable, then $\kappa \leq \Delta(X)$ (since every nonempty open subset of $X$ contains a member of each of the $\kappa$-many pairwise-disjoint dense subsets), hence the terminology "maximally resolvable".
} 
Proof. Let $\mathbb{R}$ be endowed with the standard topology.

(1) This follows immediately from the fact that every nonvoid open interval in $\mathbb{R}$ has the same cardinality as $\mathbb{R}$.

(2) We shall show that $\mathbb{R}$ is $2^{\aleph_{0}}$-resolvable. Let $\left\{r_{i}: i \in I\right\}$ be a complete set of coset representatives of $\mathbb{R}$ modulo $\mathbb{Q}$ (that is, $\mathbb{Q}+r_{i} \neq \mathbb{Q}+r_{j}$ for $i \neq j$ and for every $r \in \mathbb{R}$, there exists $i \in I$ such that $\left.\mathbb{Q}+r=\mathbb{Q}+r_{i}\right)$. Lemma 3 tells us that $|I|=2^{\aleph_{0}}$. As noted in the proof of Theorem 4, every coset $\mathbb{Q}+r_{i}$ is dense in $\mathbb{R}$. Further, any two distinct cosets are disjoint (this is a well-known fundamental result in group theory). Hence, $\left\{\mathbb{Q}+r_{i}: i \in I\right\}$ is a collection of $2^{\aleph_{0}}$ pairwise-disjoint dense subsets of $\mathbb{R}$, and (2) is verified.

We now present two equivalent formulations of a $(\star)$-space using the notion of resolvability.

Proposition 4. Let $X=(X, \mathcal{T})$ be a topological space. Then the following are equivalent.

(1) $X$ is a $(\star)$-space.

(2) $X$ is card-homogeneous and maximally resolvable.

Proof. Let $X=(X, \mathcal{T})$ be a topological space of cardinality $\kappa$. Suppose first that $X$ is a $(\star)$-space; we will show that $X$ is $\kappa$-resolvable (hence cardhomogeneous and maximally resolvable). Let $f: X \rightarrow X$ be a ( $\star$ )-function. We claim that the set $\mathcal{D}:=\left\{f^{-1}(x): x \in X\right\}$ is a collection of $\kappa$-many pairwise-disjoint dense subsets of $X$. Since $f[X]=X$, it follows that for any $x \in X, f^{-1}(x)$ is nonempty. Further, it is clear that for $x \neq y$, we have $f^{-1}(x) \cap f^{-1}(y)=\varnothing$. Now let $x \in X$ be arbitrary. It remains to show that $f^{-1}(x)$ is dense in $X$. Suppose that $O \in \mathcal{T}$ is nonempty. We will show that $f^{-1}(x) \cap O \neq \varnothing$. Since $f$ is a $(\star)$-function, we conclude that $f[O]=X$. Hence, $f(y)=x$ for some $y \in O$, and it follows that $y \in f^{-1}(x) \cap O$.

Conversely, assume that $X \neq \varnothing$ and is $\kappa$-resolvable (the case $X=\varnothing$ is trivial). Let $\mathcal{D}:=\left\{D_{i}: i<\kappa\right\}$ be a collection of $\kappa$ pairwise-disjoint dense subsets of $X$. Fix an enumeration $\left\{x_{i}: i<\kappa\right\}$ of $X$. For each $i$, define $f_{i}: D_{i} \rightarrow X$ by $f_{i}(d):=x_{i}$ for all $d \in D_{i}$. Further, let $z \in X$ be arbitrary. Now define a function $f: X \rightarrow X$ as follows.

$$
f(x)= \begin{cases}f_{i}(x), & \text { if } x \in D_{i} \text { for some } i<\kappa \\ z, & \text { otherwise. }\end{cases}
$$

We will show that $f$ is a $(\star)$-function. Note first that $f$ is well-defined since the $D_{i}$ are pairwise-disjoint. Now let $O$ be any non-empty open subset of $X$, and let $x_{i} \in X$ be arbitrary. Since $D_{i}$ is dense in $X$, there exists some

MISSOURI J. OF MATH. SCI., FALL 2014 


\section{G. OMAN}

$d \in D_{i} \cap O$. Hence by definition of $f$, we have $f(d)=f_{i}(d)=x_{i}$. This completes the proof.

We are almost ready to present the main result of this section. We first recall one final definition from the literature.

Definition 1. Let $X=(X, \mathcal{T})$ be a topological space, and let $\mathcal{B} \subseteq \mathcal{T}$. We say that $\mathcal{B}$ is a $\pi$-base of $X$ provided the following hold.

(1) $\varnothing \notin \mathcal{B}$.

(2) For every nonempty open set $O \in \mathcal{T}$, there exists an element $U \in \mathcal{B}$ such that $U \subseteq O$.

As a simple illustration of this concept, note that the collection of nonvoid open intervals with rational endpoints is a $\pi$-base for the standard topology on $\mathbb{R}$.

We are now ready to prove the main theorem of this section, which will allow us to show that quite a broad class of topological spaces are $(\star)$ spaces. This result can be proved rather quickly via the classical Disjoint Refinement Lemma (see Comfort and García-Ferreira [4, Remark 3.6(c)]). However, we present a self-contained argument.

Theorem 5. Let $X$ be a card-homogeneous topological space of infinite cardinality $\kappa$. Suppose further that there exists a $\pi$-base $\mathcal{B}$ of $X$ such that $|\mathcal{B}| \leq \kappa$. Then $X$ is a $(\star)$-space.

Proof. Let $\mathcal{S}:=\{U \times\{x\}: U \in \mathcal{B}, x \in X\}$, where $\mathcal{B}$ is a $\pi$-base such that $|\mathcal{B}| \leq \kappa$. Then $|\mathcal{S}|=\kappa$. Indeed, simply note that the function $\varphi: \mathcal{B} \times$ $X \rightarrow \mathcal{S}$ defined by $\varphi((U, x)):=U \times\{x\}$ is bijective. Thus, $|\mathcal{S}|=\mid \mathcal{B} \times$ $X \mid=\max (|\mathcal{B}|,|X|)=\kappa$.

Fix an enumeration $\left\{S_{i}: i<\kappa\right\}$ of $\mathcal{S}$. We now judiciously choose an element of $S_{i}$ for every $i<\kappa$ by recursion. Let $j<\kappa$ and suppose that for every $i<j$, we have chosen $\left(u_{i}, x_{i}\right) \in S_{i}$. We now pick an element $\left(u_{j}, x_{j}\right) \in S_{j}$ as follows. Set $A_{j}:=\left\{u_{i}: i<j\right\}$. Then note that $\left|A_{j}\right| \leq|j|<$ $\kappa$ (the final inequality follows from the fact that $\kappa$ is a cardinal, hence is not equinumerous with any smaller ordinal). By definition, $S_{j}=U_{j} \times\left\{x_{j}\right\}$ for some $U_{j} \in \mathcal{B}$ and $x_{j} \in X$. Note that $\left|U_{j}\right|=\kappa$ since $X$ is card-homogeneous of cardinality $\kappa$. As $\left|A_{j}\right|<\kappa$, there exists $\left(u_{j}, x_{j}\right) \in S_{j}$ such that $u_{j} \neq u_{i}$ for any $i<j$.

Now fix some $\alpha \in X$, and define $f: X \rightarrow X$ as follows.

$$
f(x)= \begin{cases}x_{i}, & \text { if } x=u_{i} \text { for some } i \\ \alpha, & \text { otherwise. }\end{cases}
$$

We claim that $f$ is a $(\star)$-function. Since (by construction) $u_{i} \neq u_{j}$ for any $i<j<\kappa$, it follows that $f$ is well-defined. Now let $O$ be any nonempty 
open subset of $X$, and let $y \in X$ be arbitrary. Since $\mathcal{B}$ is a $\pi$-base of $X$, there exists some $U \in \mathcal{B}$ such that $U \subseteq O$. Set $S:=U \times\{y\}$. Then $S=S_{i}$ for some $i<\kappa$, and we have $\left(u_{i}, x_{i}\right) \in S_{i}$. Thus by definition of $f$, we have $f\left(u_{i}\right)=x_{i}=y$, and the proof is complete.

We conclude the paper by addressing several natural questions which result from the previous theorem.

Question 2. How broad is the class of topological spaces which satisfy the hypotheses of Theorem 5?

This question is somewhat vague. However, we can say that there is a sense in which this class is quite broad. Before presenting our next result, we pause to define some terminology in the interest of keeping the paper as self-contained as possible.

Let $X=(X, \mathcal{T})$ be a topological space. Then $X$ is first-countable if for every $x \in X$, there exists a countable collection $B_{x}:=\left\{U_{n}(x): n \in \mathbb{Z}^{+}\right\}$ of neighborhoods of $x$ (that is, open sets containing $x$ ) such that for every neighborhood $U$ of $x$, there is a positive integer $n$ such that $U_{n}(x) \subseteq U$. Thus, in a sense, $X$ is first-countable if and only if $X$ has countable $\pi$-bases locally. We say that $X$ is second-countable if $X$ possesses a countable base for the topology (that is, a countable collection $\mathcal{B}$ of open sets such that every open set is a union of members of $\mathcal{B}$ ). $X$ is metrizable if there is a metric $d$ on $X$ such that $\mathcal{T}$ is the collection of open sets of the metric space $(X, d)$ (it is easy to show that second-countable and metrizable spaces are first-countable). $X$ is locally compact if every $x \in X$ has a compact neighborhood. $X$ is Hausdorff if for every pair of distinct elements $x$ and $y$ of $X$, there exists a neighborhood $U$ of $x$ and $V$ of $y$ such that $U \cap V=\varnothing$. $X$ is separable if $X$ has a countable dense subset. Finally, $X$ is regular if, given any nonempty closed set $F$ and any point $x$ that does not belong to $F$, there exists a neighborhood $U$ of $x$ and a neighborhood $V$ of $F$ such that $U \cap V=\varnothing$.

We now show that the class of $(\star)$-spaces is, in fact, quite broad.

Proposition 5. Let $X$ be an infinite card-homogeneous topological space. Then $X$ is a $(\star)$-space if any of the following additional properties hold.

(1) $X$ is first-countable (hence also if $X$ is second-countable or metri$z a b l e)$.

(2) $X$ is a linearly ordered space (that is, the topology on $X$ is the order topology generated from a linear order on $X)$.

(3) $X$ is a locally compact Hausdorff space.

(4) $X$ is separable, regular, and of cardinality at least $2^{\aleph_{0}}$.

Proof. We prove only that properties (1) and (2) imply that $X$ is a $(\star)$ space; we will refer the reader to the literature for (3) and (4).

MISSOURI J. OF MATH. SCI., FALL 2014 
(1) Suppose that $X$ is first-countable. For $x \in X$, let $B_{x}:=\left\{U_{n}(x)\right.$ : $\left.n \in \mathbb{Z}^{+}\right\}$be a countable base at $x$. Now consider the set $\mathcal{B}:=$ $\left\{U_{n}(x): n \in \mathbb{Z}^{+}, x \in X\right\}$. Clearly $\mathcal{B}$ is a $\pi$-basis of $X$. Further, $|\mathcal{B}| \leq\left|\mathbb{Z}^{+} \times X\right|=\max \left(\aleph_{0},|X|\right)=|X|$. Theorem 5 implies that $X$ is a $(\star)$-space.

(2) Assume now that $X$ is a linearly ordered space. Recall that the collection of all intervals of the following form yield a basis $\mathcal{B}$ for a topology on $X$ :

(i) All open intervals $(a, b):=\{x \in X: a<x<b\}$,

(ii) All half-open intervals of the form $\left[a_{0}, b\right):=\left\{x \in X: a_{0} \leq\right.$ $x<b\}$, where $a_{0}$ is the smallest element (if any) of $X$, and

(iii) All half-open intervals of the form $\left(a, b_{0}\right]:=\{x \in X: a<x \leq$ $\left.b_{0}\right\}$, where $b_{0}$ is the largest element (if any) of $X$.

Since $X$ is infinite, it follows (as in (1)) that $|\mathcal{B}| \leq|X|$. Since $\mathcal{B}$ is a basis for the topology, clearly $\mathcal{B}-\{\varnothing\}$ is a $\pi$-base of $X$. Again, Theorem 5 yields that $X$ is a $(\star)$-space.

(3)-(4) To show that spaces satisfying (in addition to being card-homogeneous) (3) and (4) are ( $\star$-spaces, it suffices by Proposition 4 to establish that they are maximally resolvable. Theorem 3.7 (a) of [4] takes care of (3); we refer the reader to Corollary 2 of Pearson [14] for (4).

Question 3. Let $X$ be an infinite card-homogeneous space. Theorem 5 shows that the existence of a $\pi$-base $\mathcal{B}$ of $X$ with $|\mathcal{B}| \leq|X|$ is sufficient to guarantee that $X$ is $a(\star)$-space. Is this condition also necessary?

The answer to this question has appeared only fairly recently in the literature. In particular, Balcerzak, Natkaniec, and Terepeta have shown that this question has a negative answer by constructing an infinite cardhomogeneous space $X$ which is maximally resolvable (hence a $(\star)$-space), yet every $\pi$-base of $X$ has cardinality strictly greater than $|X|$. The construction is a bit technical, and we do not present the details here. Instead, we refer the interested reader to Theorem 17 of [2].

We conclude this section with a consideration of the following question.

Question 4. Let $X$ be an infinite card-homogeneous space. We have seen that the existence of a $\pi$-base $\mathcal{B}$ of $X$ with $|\mathcal{B}| \leq|X|$ is sufficient to guarantee that $X$ is a $(\star)$-space, yet it is not necessary. Can this condition be dropped completely? In other words, is every infinite card-homogeneous space a $(\star)$-space?

Before presenting an answer to this question in general, we prove a proposition which yields some evidence that the answer is 'no'. 
Proposition 6. Let $X$ be an infinite set, and let $\mathcal{C}:=\{Y \subseteq X:|Y|=|X|\}$. There does not exist a function $f: X \rightarrow X$ with the property that $f[Y]=X$ for all $Y \in \mathcal{C}$.

Proof. Suppose by way of contradiction that there exists a function $f: X \rightarrow$ $X$ such that $f[Y]=X$ for all $Y \in \mathcal{C}$. Now fix some $x_{0} \in X$, and let $A:=\left\{x \in X: f(x)=x_{0}\right\}$. We claim that $\left|A^{c}\right|=\kappa$. To see this, simply note that $($ since $X \in \mathcal{C}) X=f[X]=f\left[A \cup A^{c}\right]=f[A] \cup f\left[A^{c}\right]=\left\{x_{0}\right\} \cup f\left[A^{c}\right]$. Hence,

$$
\kappa=|X|=\left|\left\{x_{0}\right\} \cup f\left[A^{c}\right]\right|=\left|f\left[A^{c}\right]\right| \leq\left|A^{c}\right| \leq \kappa .
$$

Since $\left|A^{c}\right|=\kappa$, we see that $A^{c} \in \mathcal{C}$. Thus, $f\left[A^{c}\right]=X$. But then $x_{0} \in f\left[A^{c}\right]$, and it follows that $f(x)=x_{0}$ for some $x \in A^{c}$. However, by the very definition of $A$, we have $x \in A$. This is a contradiction, and the proof is complete.

We now pause to reflect upon the previous proposition. Set $\mathcal{T}:=\mathcal{C} \cup\{\varnothing\}$. Note that if $\mathcal{T}$ happened to be a topology on $X$, then $(X, \mathcal{T})$ would be an example of an infinite card-homogeneous space which is not a $(\star)$-space. But is $\mathcal{T}$ a topology on $X$ ? Well, note that $\varnothing \in \mathcal{T}$ and $X \in \mathcal{T}$. Further, it is clear that $\mathcal{T}$ is closed under arbitrary unions. So we are close. But unfortunately $\mathcal{T}$ is not closed under finite intersections. To see this, choose $x \in X$ arbitrarily and let $A$ and $B$ be disjoint subsets of $X-\{x\}$ of cardinality $\kappa$. Then $A^{\prime}:=A \cup\{x\}$ and $B^{\prime}:=B \cup\{x\}$ are members of $\mathcal{T}$, yet $A^{\prime} \cap B^{\prime}=\{x\} \notin \mathcal{T}$.

But let's not lose all hope just yet! We can actually use the ideas of the previous proof to cook up a card-homogeneous topological space which is not a $(\star)$-space. Our saving grace is a mathematical object called an ultrafilter, which we now define.

Definition 2. Let $X$ be a nonempty set. An ultrafilter on $X$ is a collection $\mathcal{U}$ of subsets of $X$ which satisfies the following conditions.

(1) $X \in \mathcal{U}$ and $\varnothing \notin \mathcal{U}$.

(2) If $A \in \mathcal{U}$ and $B \in \mathcal{U}$, then $A \cap B \in \mathcal{U}$.

(3) If $A \in \mathcal{U}$ and $A \subseteq B \subseteq X$, then $B \in \mathcal{U}$.

(4) For every $A \subseteq X$ : either $A \in \mathcal{U}$ or $A^{c} \in \mathcal{U}$.

A simple example of an ultrafilter on a nonempty set $X$ is as follows. Let $x_{0} \in X$ be arbitrary, and let $\mathcal{U}$ be the collection of all subsets of $X$ which contain the element $x_{0}$. It is straightforward to check that conditions (1)(4) above hold, hence $\mathcal{U}$ is an ultrafilter on $X$, called a principal ultrafilter. It is easy to prove that if $X$ is finite, then every ultrafilter on $X$ is principal. If $X$ is infinite, there is always a nonprincipal ultrafilter ${ }^{8} \mathcal{U}$ on $X$. The

\footnotetext{
${ }^{8}$ Some authors call a nonprincipal ultrafilter a free ultrafilter.
} 


\section{G. OMAN}

existence of such a nonprincipal $\mathcal{U}$ depends on some weak form of the axiom of choice, though we won't go into details here (instead we refer the reader to Chapter 7 of [10]; for a more in-depth treatment of ultrafilters, see the popular text [5] by Comfort and Negrepontis). Of particular utility to us is the following simple observation which we will use shortly.

Proposition 7. Let $X$ be a nonempty set and let $\mathcal{U}$ be an ultrafilter on $X$. Then $\mathcal{T}:=\mathcal{U} \cup\{\varnothing\}$ is a topology on $X^{9}$.

Proof. By definition, $\varnothing \in \mathcal{T}$. Since $X \in \mathcal{U}$, it follows that $X \in \mathcal{T}$. As $\mathcal{U}$ is closed under supersets, it is clear that $\mathcal{T}$ is closed under arbitrary unions. Finally, because ultrafilters are closed under finite intersections, the same is true of $\mathcal{T}$.

We will soon make use of a special type of ultrafilter called a uniform ultrafilter, which is defined as follows.

Definition 3. Let $X$ be an infinite set of cardinality $\kappa$, and let $\mathcal{U}$ be an ultrafilter on $X$. Then $\mathcal{U}$ is said to be a uniform ultrafilter provided $|A|=\kappa$ for all $A \in \mathcal{U}$.

It is well-known that for every infinite set $X$, there exists a uniform ultrafilter $\mathcal{U}$ on $X$ (see [10, Theorem 7.6]). Finally, we are in position to answer Question 4.

Proposition 8. Let $\kappa$ be an infinite cardinal. There exists a card-homogeneous topological space $X$ of cardinality $\kappa$ which is not $a(\star)$-space.

Proof. Let $\mathcal{U}$ be a uniform ultrafilter on $X$, and $\mathcal{T}:=\mathcal{U} \cup\{\varnothing\}$. Since $\mathcal{U}$ is uniform, it is clear that $X=(X, \mathcal{T})$ is a card-homogeneous topological space. We will show that $X$ is not a $(\star)$-space. Suppose by way of contradiction that there exists a function $f: X \rightarrow X$ such that $f[U]=X$ for all $U \in \mathcal{U}$. Let $x_{0} \in X$ be arbitrary, and set $A:=\left\{x \in X: f(x)=x_{0}\right\}$. We claim that $A \notin \mathcal{U}$. For if so, then $f[A]=X$. But by definition of $A$, we have $f[A]=\left\{x_{0}\right\} \neq X$. We conclude that $A \notin \mathcal{U}$. Since $A \notin \mathcal{U}$, it follows from (4) of the definition of ultrafilter that $A^{c} \in \mathcal{U}$. As $f$ is a $(\star)$-function, we see that $f\left[A^{c}\right]=X$. In particular, $x_{0} \in f\left[A^{c}\right]$. Thus, $f(x)=x_{0}$ for some $x \in A^{c}$. It now follows by definition of $A$ that $x \in A$, and this is a contradiction. The proof is now complete.

Since we presented a number of results in this section, we conclude with a summary. Let $X$ be an infinite topological space. Then $X$ is a $(\star)$-space if and only if $X$ is card-homogeneous and maximally resolvable. If $X$ is card-homogeneous and has a $\pi$-base $\mathcal{B}$ of size at most $|X|$, then $X$ is a

\footnotetext{
${ }^{9}$ This topology is useful for constructing examples of so-called door spaces, that is, spaces $X$ for which every subset of $X$ is either open or closed.
} 


\section{CONVERSE OF THE INTERMEDIATE VALUE THEOREM}

$(\star)$-space. It is possible for $X$ to be a $(\star)$-space but without any $\pi$-base of size at most $|X|$. Lastly, it is possible for $X$ to be card-homogeneous yet not be a $(\star)$-space.

\section{Concluding Remarks}

The notion of maximal resolvability is well-studied in the topological literature. One might ask if "nice" necessary and sufficient conditions for a space to be maximally resolvable are known. At the present time, the answer is 'no'. For further reading on this topic, we refer the reader to [4] for a survey and to the recent paper of Comfort and $\mathrm{Hu}[6]$ for a long list of references.

Our journey from the reals to the stars has taken us from the concrete realm of decimal representations to the abstract universe of cardinalhomogenous spaces and uniform ultrafilters.

\section{AcKnowledgements}

I want to thank John Conway for his beautiful base 13 construction as well as Wistar Comfort, Todd Eisworth, Wanjun Hu, and Ronnie Pavlov for several informative conversations concerning the content of this paper; you helped to make the ride quite enjoyable. I also warmly thank the referee for the many suggestions which significantly improved the readability of this paper.

\section{REFERENCES}

[1] M. Aigner and G. Ziegler, Proofs from THE BOOK, 4th ed., Springer-Verlag, Berlin, New York, 2009.

[2] M. Balcerzak, T. Natkaniec, and M. Terepeta, Cardinal inequalities implying maximal resolvability, Comment. Math. Univ. Carolin., 46.1 (2005), 85-91.

[3] J. Ceder, On maximally resolvable spaces, Fund. Math., 55 (1964), 87-93.

[4] W. Comfort and S. García-Ferreira, Resolvability: a selective survey and some new results, Topology Appl., 74 (1996), 149-167.

[5] W. Comfort and S. Negrepontis, The Theory of Ultrafilters, Die Grundlehren der mathematischen Wissenschaften, Band 211, Springer-Verlag, New York-Heidelberg, 1974

[6] W. Comfort and W. Hu, Tychonoff expansions with prescribed resolvability properties, Topology Appl., 157.5 (2010), 839-856.

[7] H. Enderton, Elements of Set Theory, Academic Press, Harcourt Brace Jovanovich, Publishers, New York-London, 1977.

[8] S. Goldstein, D. Tausk, R. Tumulka, and N. Zanghi, What does the free will theorem actually prove?, Notices Amer. Math. Soc., 57.11 (2010), 1451-1453.

[9] T. Hungerford, Algebra, reprint of the 1974 original, Graduate Texts in Mathematics, 73, Springer-Verlag, New York-Berlin, 1980.

[10] T. Jech, Set Theory, third millennium edition, revised and expanded, Springer Monographs in Mathematics, Springer-Verlag, Berlin, 2003. 


\section{G. OMAN}

[11] D. Knuth, Surreal Numbers, Addison-Wesley Publishing Co., Reading, Mass.London-Amsterdam, 1974.

[12] S. Lang, Algebra, revised 3rd ed., Graduate Texts in Mathematics, 211, SpringerVerlag, New York, 2002.

[13] J. Munkres, Topology, 2nd ed., Prentice-Hall, Inc., Englewood Cliffs, N.J., 2000.

[14] T. Pearson, Some sufficient conditions for maximal-resolvability, Canad. Math. Bull., 14.2 (1971), 191-196.

[15] W. Rudin, Principles of Mathematical Analysis, 3rd ed., International Series in Pure and Applied Mathematics, McGraw-Hill Book Co., New York-Auckland-Düsseldorf, 1976.

[16] H. Smith, On the integration of discontinuous functions, Proc. London Math. Soc., 1.6 (1874), 140-153.

[17] H. Sohrab, Basic Real Analysis, Birkhauser Boston, Inc., Boston, MA, 2003.

[18] J. Stewart, Essential Calculus. Early Transcendentals, 2nd ed., Brooks/Cole, Cengage Learning, California, 2013.

[19] H. Teismann, Toward a more complete list of completeness axioms, Amer. Math. Monthly, 120.2 (2013), 99-114.

MSC2010: 26A15, 54C99, 54D80

Key words and phrases: Cantor set, Conway Base 13 function, coset, Intermediate Value Theorem, ultrafilter

Department of Mathematics, The University of Colorado, Colorado Springs, Colorado Springs, CO 80918

E-mail address: goman@uccs.edu 